\title{
High order stable finite difference methods for the Schrödinger equation
}

\author{
May 27, 2011
}

\author{
A. Nissen*, G. Kreiss ${ }^{\dagger}$ and M. Gerritsen ${ }^{\ddagger}$ \\ *Department of Information Technology, Uppsala University, Uppsala, \\ Sweden, anna.nissen@it.uu.se. \\ $\dagger$ Department of Information Technology, Uppsala University, Uppsala, \\ Sweden, gunilla.kreiss@it.uu.se. \\ ‡ Department of Energy Resources Engineering, Stanford University, \\ Stanford, CA, margot.gerritsen@stanford.edu.
}

\begin{abstract}
In this paper we extend the Summation-by-parts-simultaneous approximation term (SBP-SAT) technique to the Schrödinger equation. Stability estimates are derived and the accuracy of numerical approximations of interior order $2 m, m=1,2,3$, are analyzed in the case of Dirichlet boundary conditions. We show that a boundary closure of the numerical approximations of order $m$ lead to global accuracy of order $m+2$. The results are supported by numerical simulations.
\end{abstract}

\section{Introduction}

Fundamental processes at the atomic level can be described by the timedependent Schrödinger equation (TDSE), which in one dimension takes the form

$$
i \hbar \frac{\partial u}{\partial t}=-\frac{\hbar^{2}}{2 m} \frac{\partial^{2} u}{\partial x^{2}}+V u, \quad \Omega=[-L, L], \quad t \geq 0
$$


where $\hbar$ is Planck's reduced constant, $m$ is the mass of the system, and $i=\sqrt{-1}$. Equation (1.1) can be derived from the full Schrödinger equation for nuclei and electrons through a series of approximations [1], relying on the fact that electrons are light and fast compared to the nuclei. Only the movement of the nuclei is considered in (1.1), where the influence from the electrons has been modeled into the potential $V$. In equation (1.1), $x$ can denote the position of a particle (or the internuclear distance in a two-particle system), in which case $|u(x, t)|^{2}$ corresponds to the probability of the particle position $x$ at time $t$. We consider the Dirichlet boundary conditions

$$
u(-L, t)=u(L, t)=0,
$$

corresponding to infinite potential barriers which the particle cannot overcome. Figure 1 displays a time series of the probability distribution of the solution to (1.1) with $V \equiv 0$, obtained from a high-resolution numerical simulation. The initial state, a Gaussian, is propagating to the left where it hits the boundary and is reflected due to the imposed boundary conditions (1.2). The reflected pulse exhibits the dispersive character of the solution to the Schrödinger equation, as seen in the bottom right figure. The pattern that arises close to the boundary is a result of constructive and destructive interference of the superimposed plane waves constituting the Gaussian. 

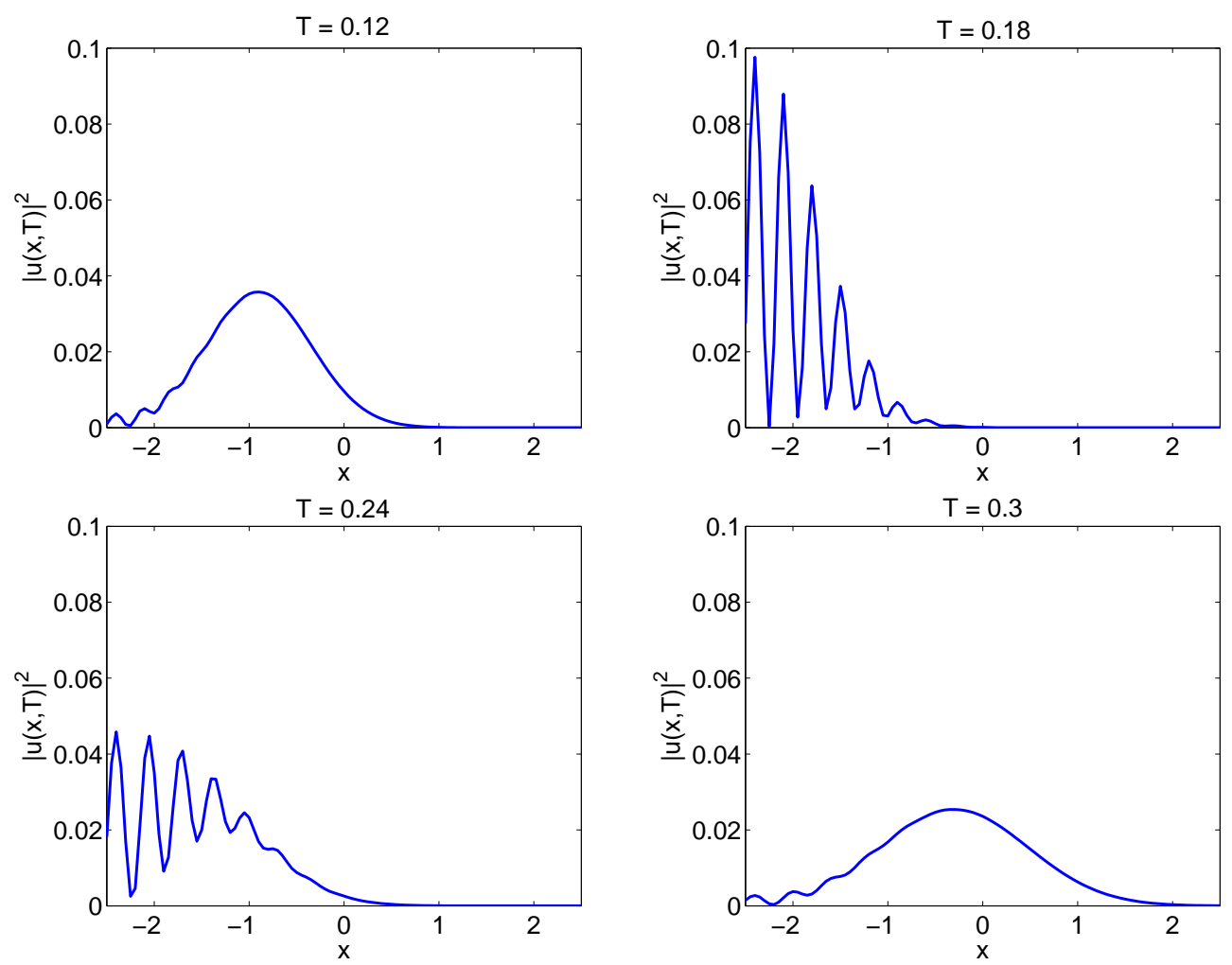

Figure 1: Time series of a Gaussian pulse traveling to the left, hitting the boundary, and being reflected back into the domain. Note that the incoming pulse in the upper left figure is more localized than the dispersed pulse in the lower right figure.

The two-dimensional TDSE

$i \hbar \frac{\partial u}{\partial t}=-\frac{\hbar^{2}}{2 m_{x}} \frac{\partial^{2} u}{\partial x^{2}}-\frac{\hbar^{2}}{2 m_{y}} \frac{\partial^{2} u}{\partial y^{2}}+V u, \quad \Omega=\left[-L_{1}, L_{1}\right] \times\left[-L_{2}, L_{2}\right], \quad t \geq 0$,

with Dirichlet boundary conditions

$$
u\left(-L_{1}, y, t\right)=u\left(L_{1}, y, t\right)=u\left(x,-L_{2}, t\right)=u\left(x, L_{2}, t\right)=0,
$$

can be interpreted as describing the internal motion of a molecule with two internal degrees of freedom, describing e.g. bond lengths of the molecule. The masses $m_{x}$ and $m_{y}$ are reduced masses of the nuclei. In a stable molecule configuration the distances between nuclei have lower and upper 
limits, and the probability distribution is in this case confined to a bounded domain $\Omega$ by the potential $V$, which can be both spatially and temporally dependent. The Dirichlet boundary conditions (1.4) can be imposed at numerical boundaries, located outside the compact support of the probability distribution. Figure 2 depicts how the probability distribution of a two-dimensional model of a Phenol molecule is located in the valley of the potential surface, which corresponds to one electronic configuration. The peaks correspond to areas with high probability. Here, the boundaries corresponding to the lower and upper limits of the internuclear distance are closed with homogeneous Dirichlet boundary conditions, whereas periodic boundary conditions is the natural choice for the rotation angle boundaries.

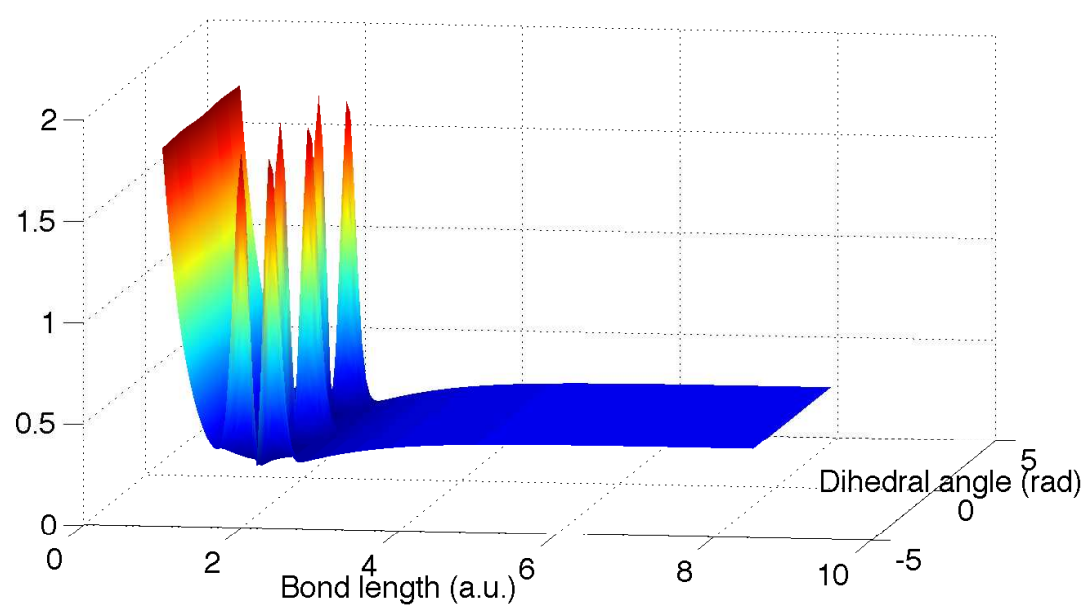

Figure 2: Probability distribution plotted together with a potential, which models one electronic configuration of a Phenol molecule.

The Schrödinger equation is commonly discretized using spectral methods that accurately capture the dispersion relation using relatively few grid points [2]. Alternatively, local approximations, such as finite difference methods can be used, but high order schemes are favored since approximations of low order typically lead to large dispersion errors.

In order to efficiently make use of computational resources, it is desirable to be able to refine the grid locally in regions where higher resolution is needed. Refinement is especially important in higher dimensions, since the total number of grid points in a static grid increases as $N^{d}$, where $d$ is the dimensionality and $N$ the number of grid points in each direction. A major drawback of spectral methods is that the global approximation 
prevents such local refinement. An additional drawback is that in a parallel framework global communication is needed in each time step, whereas in the finite difference setting only the nearest neighbor processors need to communicate. Moreover, the amount of data that needs to be communicated is $\mathcal{O}\left(N^{d}\right)$ for spectral methods and $\mathcal{O}\left(N^{d-1}\right)$ for finite difference methods.

We seek to design a high order method, with flexibility to allow local grid-refinement, that leads to an accurate and stable numerical approximation. One framework that could perhaps fulfil our requirements and be extended to the Schrödinger equation is the summation-by-partssimultaneous approximation term (SBP-SAT) method [3], which has been successfully used for many PDE's, such as the heat equation, the Euler equations and Burgers' equation. The summation-by-parts property of SBP operators leads to estimates that mimic the continuous energy estimate. In combination with weakly implemented SAT boundary treatment, stable approximations can be obtained. For a selection of the literature on the subject, we refer to $[4,5,6]$. In this paper, sections 2 and 3 contain a more detailed description of the method.

Instead of using spectral methods with exponential convergence rate, the SBP-SAT method is based on finite difference schemes, so although we may loose some accuracy, we gain flexibility and robustness.

In this paper we propose the particular form of the SAT penalty terms so that stability estimates can be derived for the Schrödinger equation. We also consider the accuracy of these approximations. The lower order schemes of the SBP operators near the boundaries lead to a lower overall convergence rate with respect to the interior stencil. However, there are results showing that the reduction of global accuracy is not as severe as the boundary approximations may suggest, see, for example, [7, 8, 9]. The approach we take on here is to explicitly investigate the determinant condition for each case, see [10], p. 514.

The main goal of this paper is to show that the SBP-SAT framework is applicable to the Schrödinger equation, and to derive the correct form of the boundary terms in this formulation. We restrict ourselves to one and two dimensions, where boundaries are closed with the Dirichlet boundary conditions described above. Extension to higher dimensions is straightforward and is together with extension to adaptive grids the topic of a forthcoming paper. A second goal is to analyze the accuracy of the approximation. We present a framework for analyzing accuracy of stable boundary closures, and apply it to our approximation. Our analysis is restricted to problems with constant coefficients and only leading order derivatives, as variable coefficients and lower order terms lead to a technically more involved analysis. In many cases, the restrictions can be removed. The premisses of the removal will be discussed to some extent. 
The structure of the paper is as follows. We start with an illustration of the second order accurate SBP-SAT method applied to the well-known usteady diffusion equation with Dirichlet boundary conditions. We derive the desirable error estimates using a new generic approach, which clearly illustrates all the steps in this process and introduces important terms and parameters. The same methodology is then applied to the Schrödinger equation, where we derive stability estimates in one and two dimensions in section 3. In section 4 we consider accuracy of the second, fourth and sixth order methods in the case of Dirichlet boundary conditions. Section 5 gives numerical evidence supporting the analysis and extending results to higher orders of accuracy. Conclusions are drawn in section 6 .

\section{Accuracy of a stable discretization of an IBVP}

Consider the linear initial boundary value problem (IBVP)

$$
\begin{array}{r}
U_{t}=D U+F(x, t), x \geq 0, t \geq 0, \\
U(x, 0)=f(x), \\
\left.B U\right|_{x=0}=0, \\
\|U(\cdot, t)\|<\infty .
\end{array}
$$

Here $B, D$ are spatial differential operators, and $F, f$ are smooth functions. We assume the problem is well posed, that it has a unique smooth solution bounded by data. See for example [10] for a precise definition. Consider a semi-discrete approximation

$$
u_{t}=\tilde{Q} u+\tilde{F}, x \geq 0, t \geq 0, u(0)=f,\|u(t)\|_{h}<\infty .
$$

In (2.5) $u, f, \tilde{F}$ denote grid functions on the grid $x_{j}=j h, j=0,1,2, \ldots$, and $\tilde{Q}$ is a difference operator into which the boundary condition (2.3) is incorporated. We assume the data is sufficiently smooth for all relevant derivatives to be bounded and continuous. Furthermore, $\|u\|_{h}$ denotes a discrete norm of the grid function $u$.

We say that the semi-discretization (2.5) is stable if the solution satisfies the energy estimate

$$
\|u(t)\|_{h} \leq K\left(\|f\|_{h}+\int_{0}^{t_{0}}\|\tilde{F}(\tau)\|_{h} d \tau\right),
$$

where $K\left(t_{0}\right)$ is independent of $f$ and $\tilde{F}$ for $t \in\left[0, t_{0}\right]$. 
We define an inner product and norm for discrete complex valued grid functions $u, v \in \mathbb{C}^{N}$,

$$
(u, v)_{P}=u^{*} P v, \quad\|u\|_{P}^{2}=u^{*} P u,
$$

where ${ }^{*}$ denotes the Hermitian transpose and $P$ is assumed to be a positive definite diagonal matrix. Note that with $P=h I$ we get the standard discrete norm.

\subsection{Accuracy by the energy estimate}

To analyze the accuracy we consider the pointwise error, defined by

$$
\tilde{\varepsilon}_{j}(t)=u_{j}(t)-U\left(x_{j}, t\right),
$$

where $U$ satisfies (2.1) and $u$ satisfies (2.5). Then

$$
\tilde{\varepsilon}_{t}=\tilde{Q} \tilde{\varepsilon}+\tilde{T}, x \geq 0, t \geq 0, \tilde{\varepsilon}(0)=0,\|\tilde{\varepsilon}\|_{h}<\infty .
$$

where $\tilde{T}$ is the truncation error, which typically depends on derivatives of the continous solution and on $h$. We say that the approximation is consistent if $\|\tilde{T}\|_{h} \rightarrow 0$ as $h \rightarrow 0$. In a bounded time interval consistency and stability can be combined to yield convergence. In particular, if $\|\tilde{T}\|_{h} \leq$ $K h^{q}$, then the convergence rate will be at least $q$.

If the truncation error $\sim h^{q}$ everywhere we cannot expect better overall convergence rate than $q$. However, in many cases the convergence rate of the truncation error is significantly lower near the boundary than in the interior. By straightforwardly applying the energy estimate the convergence rate will be governed by the lower rate at the boundary. Often numerical computations show that this convergence rate is pessimistic. We shall in the next subsections demonstrate how a more detailed analysis of (2.7) can be used to prove a sharper convergence rate result.

\subsection{Parabolic example}

To illustrate our approach we apply it to the well-known unsteady diffusion equation,

$$
U_{t}=U_{x x}+F, x \geq 0, t \geq 0
$$

with initial condition (2.2), boundary condition at $x=0$

$$
U(0, t)=0,
$$

and the requirement of a bounded $l_{2}$ norm (2.4). For this problem we assume real valued quantities. 
Consider the semi-discrete SBP-SAT approximation of (2.8), (2.9), $(2.2),(2.4)$

$$
u_{t}=Q u-\tau P^{-1} E_{0} u+\tilde{F}, t \geq 0, u(0)=f,\|u(t)\|_{h}<\infty .
$$

Here

$$
Q=P^{-1}\left(-A-E_{0} S\right), \quad A^{T}=A \geq 0,
$$

is an SBP operator of the second derivative, with interior order $2 m$ and interior width $2 m+1$, with $P=P^{T}>0$ defining the corresponding norm, see [11]. $\tau$ is here a real-valued parameter to be determined. Furthermore,

$$
S=\frac{1}{h}\left[\begin{array}{cccc}
s_{1} & s_{2} & s_{3} & \cdots \\
& 1 & & \\
& & \ddots &
\end{array}\right],
$$

where the first row of $\mathrm{S}$ approximates the first derivative. The penalty term and $Q$ also contain $E_{0}=\operatorname{diag}(1,0,0, \ldots)$. We shall use standard SBP operators for second order derivatives, given in for example [6], or [11], and for simplicity in appendix C. At $p$ points near the boundary the order of the second derivative approximation is of order $m$. The standard operators have $m=1,2,3,4$, and $p=1,4,6,8$, respectively, and correspond to diagonal norms.

The discretization (2.10) satisfies an energy estimate, i.e. is stable, if $\tau$ is chosen appropriately. To see this, introduce $R=S^{-T} A S^{-1}$, where $R$ is symmetric and positive semi-definite. Multiplying (2.10) with $u^{T} P$ and adding the transpose yields

$$
\frac{1}{2} \frac{d}{d t}\|u(t)\|_{P}^{2}=-(S u)^{T} R S u-u^{T} E_{0} S u-\tau u^{T} E_{0} u+\left(u^{T}, \tilde{F}\right)_{P} .
$$

In [6] it was shown that an estimate results due to the following lemma.

Lemma 2.1. In the second order case $R-\alpha h E_{0} \geq 0$ for any $\alpha \leq 0.4$. Note that the result is independent of $h$.

A proof of lemma 2.1 is given in appendix A. Equation (2.11) leads to

$$
\begin{array}{r}
\frac{1}{2} \frac{d}{d t}\|u(t)\|_{P}^{2}+(S u)^{T}\left(R-\alpha h E_{0}\right) S u= \\
{\left[\begin{array}{c}
u_{0} \\
(S u)_{0}
\end{array}\right]^{T} \underbrace{\left[\begin{array}{cc}
-\tau & -1 / 2 \\
-1 / 2 & -\alpha h
\end{array}\right]}_{\Gamma}\left[\begin{array}{c}
u_{0} \\
(S u)_{0}
\end{array}\right]+u^{T} P \tilde{F} .}
\end{array}
$$


If we choose $\tau \geq \frac{1}{4 \alpha h}$ then $\Gamma$ will be negative semi-definite and an estimate on the form (2.6) can be obtained. With $\tau \geq \frac{1}{4 \alpha h}$ we get by lemma 2.1

$$
\|u(t)\|_{P} \leq K\left(\|f\|_{P}+\int_{0}^{t_{0}}\|\tilde{F}(\tau)\|_{P} d \tau\right),
$$

for $t \in\left[0, t_{0}\right]$, with $K=1$. Alternatively, stability can be shown with the penalty term $\tau P^{-1} S^{T} E_{0} u$, using the methodology in [12], based on a discontinuous Galerkin formulation. In that case we do not rely on lemma 2.1. We will use this approach in section 3, where we derive stability conditions for the Schrödinger eqution. Due to the non-diffusive character of the Schrödinger equation, we cannot derive estimates using lemma 2.1.

In the $m=1$ case numerical computations show that the overall order of accuracy is 2, but a straight-forward application of the energy estimate to the error equation (2.7) yields the order 1.5. To derive a sharp estimate we shall partition the truncation error into an interior part with support away from the boundary, and a boundary part with support near the boundary. We introduce

$$
\tilde{T}=h^{m} T+h^{2 m} T^{I}, \quad T_{i} \equiv 0, i \geq p, \quad T_{i}^{I} \equiv 0, i<p .
$$

We call $T$ the boundary truncation error and $T^{I}$ the interior truncation error. They are both grid functions, and can be bounded in terms of derivatives of $U$. The error is partitioned correspondingly, $\tilde{\varepsilon}=\varepsilon+\varepsilon^{I}$. The error due to the interior truncation error, $\varepsilon^{I}$, can be estimated through the energy estimate, yielding $\left\|\varepsilon^{I}\right\|_{h} \leq K h^{2 m}$. For $\varepsilon$, the error due to the boundary truncation error, we will use another technique.

\subsubsection{Accuracy by Laplace transform}

In this part of the paper we will estimate the error due to the boundary truncation error, $\varepsilon$, in (2.7). In the case $m=1$ there is an $\mathcal{O}(h)$ error in only one point. We have,

$$
\begin{array}{r}
\frac{\partial \varepsilon_{0}}{\partial t}=D_{+} D_{-} \varepsilon_{1}-\frac{\tilde{\tau}}{h^{2}} \varepsilon_{0}+h T_{0}, \\
\frac{\partial \varepsilon_{j}}{\partial t}=D_{+} D_{-} \varepsilon_{j}, j=1,2 \ldots, \\
\|\varepsilon(t)\|_{h}<\infty,
\end{array}
$$

where $\tilde{\tau}=2 h \tau$ is independent of $h$. The operators $D_{+}, D_{-}$are defined as

$$
D_{+} u_{j}=\frac{u_{j+1}-u_{j}}{h}, \quad D_{-} u_{j}=\frac{u_{j}-u_{j-1}}{h},
$$


so that $D_{+} D_{-}$is the standard central second order stencil approximating the second derivative. Here $T_{0}$ is bounded in terms of spatial derivatives of the solution $U$. The precise estimate is of the form

$$
\left|T_{0}(t)\right| \leq \frac{1}{12} \max _{0 \leq x \leq h}\left|U_{x x x}(x, t)\right| .
$$

After Laplace transformation, where we introduce $s$ as dual variable, and multiplication by $h^{2}$ we have

$$
\begin{array}{r}
s h^{2} \hat{\varepsilon}_{0}=\hat{\varepsilon}_{0}-2 \hat{\varepsilon}_{1}+\hat{\varepsilon}_{2}-\tilde{\tau} \hat{\varepsilon}_{0}+h^{3} \hat{T}_{0}, \\
s h^{2} \hat{\varepsilon}_{j}=\hat{\varepsilon}_{j-1}-2 \hat{\varepsilon}_{j}+\hat{\varepsilon}_{j+1}, j=1,2, \ldots, \\
\|\hat{\varepsilon}\|_{h}<\infty .
\end{array}
$$

This is a difference equation which we can solve. The general solution is on the form $\kappa^{j}$, where $\kappa$ satisfies the characteristic equation arising from (2.14),

$$
\kappa^{2}-(\tilde{s}+2) \kappa+1=0 .
$$

We say that a root $\kappa$ is admissible if it satisfies (2.15), i.e. $|\kappa|<1$. The roots of (2.16) will be continuous functions of $\tilde{s} \equiv s h^{2}$ and have properties given by lemma 2.2 .

Lemma 2.2. Consider the characteristic equation (2.16) for $\operatorname{Re}(\tilde{s})>0$.

The roots are

$$
\begin{array}{ll}
\kappa_{1}(\tilde{s})=1+\frac{\tilde{s}}{2}+\sqrt{\left(\frac{\tilde{s}}{2}\right)^{2}+\tilde{s}}, & \left|\kappa_{1}\right|>1, \\
\kappa_{2}(\tilde{s})=1+\frac{\tilde{s}}{2}-\sqrt{\left(\frac{\tilde{s}}{2}\right)^{2}+\tilde{s}}, & \left|\kappa_{2}\right|<1,
\end{array}
$$

i.e. $\kappa_{2}$ is the only admissible root. In the vicinity of $\tilde{s}=0$ the admissible root satisfies

$$
\begin{array}{r}
\kappa_{2}(\tilde{s})=1-\sqrt{\tilde{s}}+\mathcal{O}(|\tilde{s}|), \\
\frac{1}{1-\left|\kappa_{2}(\tilde{s})\right|^{2}} \leq \frac{1+\mathcal{O}(\sqrt{|\tilde{s}|})}{\sqrt{2|\tilde{s}|}}
\end{array}
$$

Proof: Equations (2.17) and (2.18) are standard formulas, where the branch cut for the square root is along the negative axis. Equation (2.19) follows by Taylor expansion. Equation (2.20) can be derived by expressing 
$\tilde{s}=|\tilde{s}| e^{i \theta},|\theta| \leq \frac{\pi}{2}$. We get from $(2.19)$ that $\kappa_{2}(\tilde{s})=1-\sqrt{|\tilde{s}|} e^{i \theta / 2}+\mathcal{O}(|\tilde{s}|)$. This yields

$$
\begin{aligned}
\left|\kappa_{2}(\tilde{s})\right|^{2}=\left(1-\sqrt{|\tilde{s}|} \cos \frac{\theta}{2}\right)^{2} & +\left(\sqrt{|\tilde{s}|} \sin \frac{\theta}{2}\right)^{2}+\mathcal{O}(|\tilde{s}|) \\
= & 1-2 \sqrt{|\tilde{s}|} \cos \frac{\theta}{2}+\mathcal{O}(|\tilde{s}|)
\end{aligned}
$$

and since $\cos \frac{\theta}{2} \geq \frac{1}{\sqrt{2}}$

$$
\frac{1}{1-\left|\kappa_{2}(\tilde{s})\right|^{2}}=\frac{1}{2 \sqrt{|\tilde{s}|} \cos \frac{\theta}{2}+\mathcal{O}(|\tilde{s}|)} \leq \frac{1+\mathcal{O}(\sqrt{|\tilde{s}|})}{\sqrt{2|\tilde{s}|}}
$$

The general solution of (2.13)-(2.15) is

$$
\hat{\varepsilon}_{j}=\sigma \kappa_{2}^{j}, j=0,1,2, \ldots
$$

The coefficient $\sigma$ is determined from (2.13). By using (2.21) and the fact that $\kappa_{2}$ satisfies the characteristic equation (2.16) we can rewrite (2.13) as

$$
\underbrace{\left(\tilde{s}+\tilde{\tau}-\tilde{s} \kappa_{2}\right)}_{C(\tilde{s})} \sigma=h^{3} \hat{T}_{0}
$$

where $\tilde{\tau} \geq \frac{h}{4 \alpha P_{11}}$ is independent of $h$.

Note that it is straight-forward to extend the definition of $C(\tilde{s})$ from $\operatorname{Re}(\tilde{s})>0$ to $\operatorname{Re}(\tilde{s}) \geq 0$, yielding a continuous function. We remark that if $C(\tilde{s}) \neq 0$ for all $\operatorname{Re}(\tilde{s}) \geq 0$ the approximation is said to be boundary stable, or to satisfy the Kreiss condition [10]. Further, by stability (2.12) we can be sure $C(\tilde{s}) \neq 0$ for all $\operatorname{Re}(\tilde{s})>0$. In particular we note that in the vicinity of $\tilde{s}=0$ we have by (2.19) that

$$
C(\tilde{s})=\tilde{\tau}+\mathcal{O}\left(|\tilde{s}|^{1.5}\right),
$$

which is uniformly bounded away from zero in a neighborhood of $\tilde{s}=0$. In this case a straight-forward calculation shows that $|C(\tilde{s})|^{-1}$ is uniformally bounded for all $\operatorname{Re}(\tilde{s}) \geq 0$.

However, for the analysis of accuracy we only need to consider a neighborhood of $\tilde{s}=0$. The motivation is as follows: We are seeking an asymptotic accuracy result. The asymptotic behavior will only be seen when all relevent components of the solution are well resolved. This corresponds to the relevant part of the truncation error having bounded support in the $s$-plane. We shall only consider the well-resolved part, and therefore assume there is some constant $K_{s}$ such that $\hat{T}(s) \equiv 0$ for $|s| \geq K_{s}$. Thus 
$\hat{T}(\tilde{s}) \equiv 0$ for $|\tilde{s}| \geq \delta, \delta=h^{2} K_{s}$, which shows that we only need to consider a small neighborhood of $\tilde{s}=0$ when estimating the asymptotic effect of the boundary truncation error.

By (2.23), $|C(0)|^{-1} \mid \leq 1 / \tilde{\tau}$, and by continuity there exists a $\delta>0$ such that $|\tilde{s}| \leq \delta$ implies $|C(\tilde{s})|^{-1} \mid \leq 2 / \tilde{\tau}$. By $(2.22)$ we have a good $\tilde{s}$ independent bound for $\sigma$ when $|\tilde{s}| \leq \delta$. For $h$ sufficiently small, $h^{2} K_{s} \leq \delta$, and $\sigma \equiv 0$ when $|\tilde{s}|>\delta$. Since $\left|\kappa_{2}\right| \leq 1,\left|\hat{\varepsilon}_{j}\right| \leq|\sigma|$ at all grid points. Thus an estimate in physical space follows by Parseval's relation:

$$
\begin{array}{r}
\int_{0}^{\infty}\left|\varepsilon_{j}\right|^{2} d t=\frac{1}{2 \pi} \int_{-\infty}^{\infty}\left|\hat{\varepsilon}_{j}(\cdot, \eta+i \xi)\right|^{2} d \xi \\
\leq h^{6} \frac{2}{\tilde{\tau}^{2} \pi} \int_{-\infty}^{\infty}\left|\hat{T}_{0}\right|^{2} d \xi=h^{6} \frac{4}{\tilde{\tau}^{2}} \int_{0}^{\infty}\left|T_{0}\right|^{2} d t .
\end{array}
$$

By arguing that the future cannot affect the past the upper limit of both time integrals can be replaced by any $t_{0}>0$. A bound on $\|\varepsilon\|_{h}$ follows from (2.20) since

$$
\|\hat{\varepsilon}\|_{h}^{2}=\sum_{j=0}^{\infty}\left|\hat{\varepsilon}_{j}\right|^{2} h=\sigma^{2} h \sum_{j=0}^{\infty}\left|\kappa_{2}(\tilde{s})^{j}\right|^{2}=\frac{\sigma^{2} h}{1-\left|\kappa_{2}(\tilde{s})\right|^{2}} \leq C \frac{\sigma^{2} h}{\sqrt{\tilde{s}}} .
$$

By Parseval's relation we have

$$
\begin{array}{r}
\int_{0}^{\infty}\|\varepsilon\|_{h}^{2} d t=\frac{1}{2 \pi} \int_{-\infty}^{\infty}\|\hat{\varepsilon}(\cdot, \eta+i \xi)\|_{h}^{2} d \xi \leq \frac{\tilde{C}}{2 \pi} \int_{-\infty}^{\infty} \frac{h^{7}\left|\hat{T}_{0}\right|^{2}}{\tilde{\tau}^{2} \sqrt{|\tilde{s}|}} d \xi \\
\leq h^{6} \frac{K}{2 \pi} \int_{-\infty}^{\infty} \max _{0 \leq x \leq h}\left|\hat{U}_{x}(x, \eta+i \xi)\right|^{2} d \xi=h^{6} K \int_{0}^{\infty} \max _{0 \leq x \leq h}\left|U_{x}(x, t)\right|^{2} d t .
\end{array}
$$

In the second inequality we have used that $\left|\hat{U}_{x x x}\right|=|s|\left|\hat{U}_{x}\right|$ and that $h|s|^{2} / \sqrt{|\tilde{s}|}=|s|^{1.5}$ is bounded. This proves that for this case, asymptotically the boundary error converges to zero with order 3 .

\subsection{Accuracy in general}

In a more general case there will be a quadratic matrix $C(\tilde{s})$ whose inverse needs to be bounded. This is the same matrix as appears in the determinant condition (see [10], chapter 12). In this section we present the steps of the accuracy analysis in the general case.

Consider a general IBVP (2.1) discretized by (2.5). We make the following assumptions.

\section{Assumption}

1. At the $p<\infty$ points closest to the boundary the truncation error is $h^{m} T_{j}, j=0,1, \ldots, p-1$. 
2. Sufficiently far away from the boundary the difference operator has constant coefficients and is stable when applied to a periodic problem.

3. There is an integer $\ell>0$ such that $\bar{Q}=h^{\ell} \tilde{Q}$ is independent of $h$.

The second assumption requires that the coefficients of the problem are constant, while the last assumption corresponds to $\tilde{Q}$ approximating $\ell^{\text {th }}$ order derivatives, and that there are no lower order terms.

Equation (2.7) is divided into two parts considered separately, one equation for the error due to interior truncation error $T^{I}, \varepsilon^{I}$, and one equation for the error due to the boundary truncation error, $\varepsilon$. $\varepsilon^{I}$ is estimated through the stability estimate, straight-forwardly yielding convergence rate $2 m$. The effect of the boundary truncation error $h^{m} T_{j}$ is analyzed by considering (2.7) with $\tilde{T}$ replaced by $h^{m} T$ and $\tilde{\varepsilon}$ replaced by $\varepsilon$. After Laplace transformation and multiplication with $h^{\ell}$ we have

$$
\tilde{s} \hat{\varepsilon}_{t}=\bar{Q} \hat{\varepsilon}+h^{m+\ell} \hat{T}, \tilde{s}=s h^{\ell} .
$$

Consider this difference equation in the right half plane $\operatorname{Re}(\tilde{s})>0$. There are solutions of the form $\kappa^{j}$ where $|\kappa|$ are the admissible roots of a characteristic equation, i.e. the roots for which $|\kappa|<1$. The assumption of stability of the interior difference operator ensures that the number of admissible roots is constant in the right half plane $\operatorname{Re}(\tilde{s})>0$. The general solution will be

$$
\hat{\varepsilon}_{j}=\sum_{n=1}^{r} \sigma_{n}\left(\kappa_{n}\right)^{j-N}, \quad j=N, N+1, N+2, \ldots
$$

Here $\left(\kappa_{1}, \ldots, \kappa_{r}\right)$ are the admissible roots of the characteristic equation, and $N$ depends on how many points $b$ are affected by the boundary error or the penalty terms, and on how many admissible roots there are. There will also be $N$ free parameters $\hat{\varepsilon}_{0}, \ldots, \hat{\varepsilon}_{N-1}$ in the solution, where $N=b-m$. Inserting (2.26) and the free parameters into (2.25) yields a linear system

$$
C(\tilde{s}) \Sigma=h^{m+\ell} \hat{T}_{B}
$$

Here $\Sigma=\left(\sigma_{1}, \ldots, \sigma_{r}, \hat{\varepsilon}_{0}, \ldots, \hat{\varepsilon}_{N-1}\right)^{T}$ and $\hat{T}_{B}$ contains the $b$ first components of $\hat{T}$. We are now ready to formulate and prove the following theorem.

Theorem 2.3. Consider a discretization (2.5) of (2.1) satisfying an energy estimate (2.6), and the assumptions 1-3 stated above. We also assume that the Laplace transform of the boundary truncation error has bounded support, that is for some $K_{s}\left|\hat{T}_{j}(s)\right| \equiv 0, j=0,1, \ldots p-1$ for $|s| \geq K_{s}$. If $C(\tilde{s})$ is uniformly invertible near the origin then the effect of the boundary 
error will decay as $h^{m+\ell}$. More specifically, if there exists $K_{c}<\infty, \delta>0$ such that $\left|C(\tilde{s})^{-1}\right| \leq K_{c}$ for all $\operatorname{Re}(\tilde{s}) \geq 0,|\tilde{s}| \leq \delta$, then the solution of (2.25) satisfies

$$
\int_{0}^{t_{0}}\left|\varepsilon_{j}\right|^{2} d t \leq h^{2(m+\ell)} K_{c}^{2} \int_{0}^{t_{0}}|T|^{2} d t, \quad j=0,1,2, \ldots
$$

The proof follows exactly as in the parabolic case. We remark that usually it is sufficient to require $C(0)$ to be invertible. By continuity the invertibility is valid in a neighborhood of $\tilde{s}=0$. As $\left|\kappa_{n}\right| \leq 1$ a pointwise bound of the Laplace transformed error follows. Using a Parseval equality as in (2.24) this bound leads to a time-integral estimate in physical space, which concludes the proof.

To ensure that we only consider well resolved solutions we have assumed bounded support of the solution (and of $\hat{T}$ ) in Laplace space. In practice we need the resolution to be sufficient for asymptotic error behavior. In many cases the result extends to variable coefficient problems by the frozen coefficient approach, see [10]. Also lower order terms can in general be allowed. They are expected to cause only $\mathcal{O}(h)$ perturbations to the matrix $C$, and are therefore of no significance for the analysis.

\section{Stability of the semi-discrete Schrödinger equation}

We consider the Schrödinger equation in the simplest possible setting, one space dimension without potential,

$$
U_{t}=i U_{x x}+F, x \in \Omega, t \geq 0, U(x, 0)=f(x) .
$$

We will consider half-plane problems with a Dirichlet boundary condition:

$$
\Omega=[0, \infty), U(0, t)=0,\|U\|<\infty .
$$

We shall also consider the two-dimensional Schrödinger equation,

$$
U_{t}=i U_{x x}+i U_{y y}+F, x, y \in \Omega, t \geq 0, U(x, y, 0)=f(x, y),
$$

on a half-plane with Dirichlet boundary condition:

$$
\Omega=[0, \infty) \times(-\infty, \infty), U(0, y, t)=0,\|U\|<\infty .
$$




\subsection{Analysis of stability for the Schrödinger equation}

Consider the semi-discrete SBP-SAT problem corresponding to (3.1), (3.2):

$$
u_{t}=i Q u-\tau P^{-1} S^{T} E_{0} u+F, t \geq 0, u(0)=f,\|u(t)\|_{h}<\infty .
$$

Here $u, f, F$ are grid functions on the grid $x_{j}=j h, j=0,1,2, \ldots$, and $Q$ is a "standard" SBP operator of the second derivative, with interior order $2 m$ and interior width $2 m+1$, with $P$ defining the corresponding norm [11]. As in the parabolic case we have $Q=P^{-1}\left(-A-E_{0} S\right)$, with $A^{T}=A>0 . E_{0}$ and $S$ are defined in section 2.2. At $p$ points near the boundary the order of the second derivative approximation is $m$. We recall that the standard SBP operators have $m=1,2,3,4$, and $p=1,4,6,8$, respectively, and correspond to diagonal norms. Here, $\tau$ is assumed to be a complex-valued parameter to be determined.

We also consider the semi-discrete problem in two space dimensions corresponding to (3.3), (3.4),

$$
\begin{array}{r}
\boldsymbol{u}_{t}=\left\{\left(i Q_{x}-\tau P_{x}^{-1} S_{x}^{T} E_{0, x}\right) \otimes I_{y}+I_{x} \otimes i Q_{y}\right\} \boldsymbol{u}+\boldsymbol{F}, \\
t \geq 0, \boldsymbol{u}(0)=\boldsymbol{f},\|\boldsymbol{u}(t)\|_{h} \leq \infty
\end{array}
$$

where the solution vector $\boldsymbol{u}$ is organized with elements initially changing along the $y$-direction. $I_{x}$ and $I_{y}$ are identity matrices and $E_{0, x}=$ $\operatorname{diag}(1,0,0, \ldots)$. We can show that the discretizations (3.5) and (3.6) satisfies energy estimates with non-decaying energy if $\tau$ is chosen appropriatly. The energy conservation is an important property of the numerical scheme, since the $l_{2}$ norm is conserved for the continuous equation.

Theorem 3.1. Consider the right half plane problems in one and two space dimensions, (3.1) and (3.3), with homogeneous Dirichlet boundary conditions, discretized by the SBP-SAT discretizations (3.5) and (3.6), respectively. If $\tau$ is chosen as $\tau=i$ then (3.5) and (3.6) are stable by the estimates

$$
\begin{aligned}
& \|u(t)\|_{P} \leq\|f\|_{P}+\int_{0}^{t_{0}}\|F(\tau)\|_{P} d \tau, \\
& \|\boldsymbol{u}(t)\|_{P} \leq\|\boldsymbol{f}\|_{P}+\int_{0}^{t_{0}}\|\boldsymbol{F}(\tau)\|_{P} d \tau,
\end{aligned}
$$

in any time interval $t \in\left[0, t_{0}\right]$. 
Proof: Multiplying (3.5) with $u^{*} P$ and adding the Hermitian transpose leads to the energy estimate

$$
\frac{d}{d t}\|u\|_{P}^{2}=\left[\begin{array}{c}
u_{0} \\
(S u)_{0}
\end{array}\right]^{*}\left[\begin{array}{cc}
0 & -i-\tau^{*} \\
i-\tau & 0
\end{array}\right]\left[\begin{array}{c}
u_{0} \\
(S u)_{0}
\end{array}\right]+u^{*} P F+F^{*} P u .
$$

By choosing $\tau=i$, equation (3.5) satisfies the estimate

$$
\frac{d}{d t}\|u\|_{P}^{2}=2\|u\|_{P} \frac{d}{d t}\|u\|_{P}=u^{*} P F+F^{*} P u \leq 2\|u\|_{P}\|F\|_{P} .
$$

Dividing by $2\|u\|_{P}$ and integrating in time leads to (3.7). The energy estimate of the two-dimensional problem is completely analogous to the one-dimensional energy estimate, and thus leads to the same penalty parameter for the right half-plane problem. Multiplying (3.6) with $\boldsymbol{u}^{*} P$, where $P=P_{x} \otimes P_{y}$, and adding the transpose leads to

$$
\begin{array}{r}
\frac{d}{d t}\|\boldsymbol{u}\|_{P}^{2}=\boldsymbol{u}^{*} P \boldsymbol{u}_{t}+\boldsymbol{u}_{t}^{*} P \boldsymbol{u} \\
=\boldsymbol{u}^{*}\left(\left\{(-\tau+i) S_{x}^{T} E_{0, x}+\left(-\tau^{*}-i\right) E_{0, x} S_{x}\right\} \otimes P_{y}\right) \boldsymbol{u}+\boldsymbol{u}^{*} P F+F^{*} P \boldsymbol{u} .
\end{array}
$$

The estimate (3.8) is obtained by choosing the penalty $\tau=i$, and integrating in time.

If we instead consider the left half-plane problems, stability for the one- and two-dimensional problems are obtained by (3.7) and (3.8) for the corresponding penalty $\tau=-i$.

Remark: The stability estimates (3.7) and (3.8) are valid for equations (1.1) and (1.3) also in the case of a non-zero potential $V$.

\section{Analysis of accuracy of the SBP-SAT bound- ary treatment}

We will analyze the one dimensional problem. Assuming that the continuous problem has a smooth solution $U(x, t)$ we can derive an equation for the pointwise error,

$$
\tilde{\varepsilon}_{j}(t)=U\left(x_{j}, t\right)-u_{j}(t)
$$

where the truncation error appears as a forcing term. In the Dirichlet case (3.5) we shall analyze the approximations with interior order $2 m=2,4,6$. The error equation is of the form

$$
\tilde{\varepsilon}_{t}=i Q \tilde{\varepsilon}-\tau P^{-1} S^{T} E_{0} \tilde{\varepsilon}+\tilde{T}^{(m)}, t \geq 0, \tilde{\varepsilon}(0)=0,\|\varepsilon(t)\|_{h}<\infty .
$$


The first $p$ components of $\tilde{T}^{(m)}$ are at worst of order $\mathcal{O}\left(h^{m}\right)$ while all other components are of order $\mathcal{O}\left(h^{2 m}\right)$. We introduce $T^{(m)}$ and $T^{(I, m)}$ such that

$$
\begin{array}{r}
\tilde{T}^{(m)}=h^{m} T^{(m)}+h^{2 m} T^{(I, m)}, \\
T_{j}^{(m)} \equiv 0, j \geq p, \\
T_{j}^{(I, m)} \equiv 0, j<p .
\end{array}
$$

Here $T^{(m)}$ and $T^{(I, m)}$ depend on derivatives of $U$, and $p$ is the number of points in the vicinity of the boundary where special stencils are used. In the analysis of $\varepsilon$ we will consider the Laplace transform of (4.1),

$$
s \hat{\varepsilon}=i Q \hat{\varepsilon}-\tau P^{-1} S^{T} E_{0} \hat{\varepsilon}+h^{m} \hat{T}^{(m)},\|\hat{\varepsilon}\|_{h}<\infty,
$$

for $\operatorname{Re}(s)>0$. Sufficiently far away from the boundary (4.3) is of the form

$$
\begin{aligned}
& m=1: \quad s \hat{\varepsilon}_{k}=i D_{+} D_{-} \hat{\varepsilon}_{k}, \\
& m=2: \quad s \hat{\varepsilon}_{k}=i\left(D_{+} D_{-}-\frac{h^{2}}{12}\left(D_{+} D_{-}\right)^{2}\right) \hat{\varepsilon}_{k} \\
& m=3: \quad s \hat{\varepsilon}_{k}=i\left(D_{+} D_{-}-\frac{h^{2}}{12}\left(D_{+} D_{-}\right)^{2}+\frac{h^{4}}{90}\left(D_{+} D_{-}\right)^{3}\right) \hat{\varepsilon}_{k} .
\end{aligned}
$$

We will prove the following theorem.

Theorem 4.1. Consider (3.5) for $m=1,2,3$ and assume the continuous solution is smooth, with all relevant derivatives bounded in the norms $\|\cdot\|_{h}$ and $|\cdot|_{\infty}$ in all intervals $0 \leq t \leq t_{0}$. We also assume that the boundary truncation error, defined by (4.2), has Laplace transform with bounded support, that is for some $K_{s}\left|\hat{T}_{j}^{(m)}(s)\right| \equiv 0, j=0,1, \ldots, p-1$ for $|s| \geq K_{s}$. The error, partitioned into boundary error $\varepsilon$ and interior error $\varepsilon^{I}$ corresponding to $T^{(m)}$ and $T^{(I, m)}$, satisfies

$$
\begin{array}{r}
\left\|\varepsilon^{I}(t)\right\|_{h} \leq h^{2 m} K_{1}^{(m)}\left(t_{0}\right), \\
\sqrt{\int_{0}^{t_{0}}\left|\varepsilon_{j}(t)\right|^{2} d t} \leq h^{2\left[\frac{m}{2}\right]+3} K_{2}^{(m)}\left(t_{0}\right), \quad j=0,1, \ldots, \\
\sqrt{\int_{0}^{t_{0}}\|\varepsilon(t)\|_{h}^{2} d t} \leq h^{m+2} K_{3}^{(m)}\left(t_{0}\right) .
\end{array}
$$

Here $K_{1}, K_{2}, K_{3}$ depend only on $U$ and its derivatives, and on the time interval. $\left[\frac{m}{2}\right]$ denotes the integer part of $\frac{m}{2}$.

Proof: Consider first (4.1) with only the interior part of the truncation error as forcing. From the smoothness assumptions $\left\|T^{(I, m)}\right\|^{2}$ is bounded, and the estimate (4.7) follows directly by applying theorem 3.1. 
To derive (4.8) we shall use theorem 2.3, while (4.9) will follow by a simple extension. We will derive the matrix $C(\tilde{s})$ associated with (4.3), and show that it is invertible in a vicinity of $\operatorname{Re}(\tilde{s})=0$. The estimates are proven to hold in sections 4.1, 4.2 and 4.3 for second, fourth, and sixth order of accuracy, respectively. We will need lemma 4.2 and corollary 4.3 for the proof of the theorem.

Lemma 4.2. Let $\tilde{s}=s h^{2}$ and consider the characteristic equations corresponding to (4.4),(4.5) and (4.6). As long as $\operatorname{Re}(\tilde{s})>0$ there are no characteristic roots with $|\kappa|=1$. For $m=1,2,3$ the number of admissible roots, satisfying $|\kappa|<1$ for $\operatorname{Re}(\tilde{s})>0$, is $1,2,3$, respectively. In the vicinity of $\tilde{s}=0$ they are given by

$$
\begin{gathered}
m=1: \quad \kappa_{1}=1+\frac{-1+i}{\sqrt{2}} \sqrt{\tilde{s}}+\mathcal{O}(\tilde{s}), \\
m=2: \quad \kappa_{1}=1-\frac{1+i}{\sqrt{2}} \sqrt{\tilde{s}}+\mathcal{O}(\tilde{s}), \kappa_{2}=7-4 \sqrt{3}+\mathcal{O}(\tilde{s}), \\
m=3: \quad \kappa_{1}=1-\frac{1+i}{\sqrt{2}} \sqrt{\tilde{s}}+\mathcal{O}(\tilde{s}), \kappa_{2}=\kappa_{2}^{(0)}+\mathcal{O}(\tilde{s}), \\
\kappa_{3}=\kappa_{3}^{(0)}+\mathcal{O}(\tilde{s}), \kappa_{2}^{(0)} \approx 0.05189+0.08009 i, \kappa_{3}^{(0)} \approx 0.05189-0.08009 i .
\end{gathered}
$$

Proof: The first claim is proven by contradiction. Similar proofs are found in for example [10]. Consider solving a periodic Cauchy problem. It is trivial to show that the standard central difference operator of order $2 m$, denoted by $Q_{0}^{(2 m)}$ (given by the right hand side operators of (4.4)(4.6) for $m=1,2,3$ ), yields a stable semi-discretization, with the symbol of the spatial operator satisfying $\operatorname{Re}\left(\hat{Q}_{0}^{(2 m)}(\xi)\right)=0$ for all $\xi$. Assume the corresponding characteristic equation has a root $|\kappa|=1=\left|e^{i \theta}\right|$ for some $\operatorname{Re}(\tilde{s})>0$. This implies

$$
s=\hat{Q}_{0}^{(2 m)}(h \theta) .
$$

This is a contradiction since the left hand side has a strictly positive real part while the right hand side is purely imaginary.

In the second order case the characteristic equation corresponding to (4.4) is

$$
\kappa^{2}+(i \tilde{s}-2) \kappa+1=0,
$$

with roots

$$
\kappa_{1}=1-i \frac{\tilde{s}}{2}+\sqrt{-\left(\frac{\tilde{s}}{2}\right)^{2}-i \tilde{s}}, \quad \kappa_{2}=1-i \frac{\tilde{s}}{2}-\sqrt{-\left(\frac{\tilde{s}}{2}\right)^{2}-i \tilde{s}}
$$


As the number of admissible roots is constant for all $\operatorname{Re}(\tilde{s})>0$, they can be determined from some extreme value. In the vicinity of $\tilde{s}=0$ we find by Taylor expansion that there is one admissible root, given by (4.10).

In the fourth order case the characteristic equation is

$$
-\kappa^{4}+16 \kappa^{3}+(i 12 \tilde{s}-30) \kappa^{2}+16 \kappa-1=0 .
$$

At $\tilde{s}=0$ (4.13) can be factorized into

$$
(\kappa-1)^{2}\left(-\kappa^{2}+14 \kappa-1\right)=0,
$$

with a double root at $\kappa=1$, and two single roots, one of which is admissable, and given by the second expression in (4.11). By perturbation analysis we find that away from $\tilde{s}=0$ the double root separates into one admissible and one inadmissible. In particular we find that the admissible root is given by the first expression in (4.11).

In the sixth order case (we omit the details) one finds that the characteristic equation at $\tilde{s}=0$ can be factorized into

$$
(\kappa-1)^{2}\left(2 \kappa^{4}-23 \kappa^{3}+222 \kappa^{2}-23 \kappa+2\right)=0,
$$

which has three admissible roots. Near $\tilde{s}=0$ they are given by (4.12).

Corollary 4.3. Consider $|s| \leq K, \operatorname{Re}(s) \geq \eta>0$. For $m=1,2,3$ the admissible roots $\kappa_{1}$ satisfies

$$
1-\left|\kappa_{1}(\tilde{s})\right|^{2} \geq \frac{\eta}{K} \sqrt{|\tilde{s}|}(1+\sqrt{|\tilde{s}|}) .
$$

Proof: Write $\tilde{s}=|\tilde{s}| e^{i \theta}$ with $|\theta| \leq \pi / 2-\alpha, \alpha>0, \sin (\alpha) \geq \eta / K$. By (4.10) we can express $\kappa_{1}(\tilde{s})=1+\sqrt{|\tilde{s}|} e^{i\left(\frac{\theta}{2}+\frac{3 \pi}{4}\right)}+\mathcal{O}(\tilde{s})$ and by trigonometric manipulations we get

$$
\begin{aligned}
& 1-|\kappa(\tilde{s})|^{2}=-2 \sqrt{|\tilde{s}|} \cos \left(\frac{\theta}{2}+\frac{3 \pi}{4}\right)+\mathcal{O}(|\tilde{s}|) \\
& \geq 2 \sqrt{|\tilde{s}|} \cos \left(\frac{\pi}{2}-\frac{\alpha}{2}\right)+\mathcal{O}(|\tilde{s}|) \\
& \geq \sqrt{|\tilde{s}|} \sin (\alpha)+\mathcal{O}(|\tilde{s}|) \geq \frac{\eta}{K} \sqrt{|\tilde{s}|}(1+\mathcal{O}(\sqrt{|\tilde{s}|})) .
\end{aligned}
$$

Starting from (4.11), (4.12) in the fourth and sixth order cases we have $\kappa_{1}(\tilde{s})=1+\sqrt{|\tilde{s}|} e^{i\left(\frac{\theta}{2}-\frac{3 \pi}{4}\right)}+\mathcal{O}(\tilde{s})$ and similarly get

$$
\begin{array}{r}
1-|\kappa(\tilde{s})|^{2}=-2 \sqrt{|\tilde{s}|} \cos \left(\frac{\theta}{2}-\frac{3 \pi}{4}\right)+\mathcal{O}(|\tilde{s}|) \\
\geq 2 \sqrt{|\tilde{s}|} \cos \left(\frac{\pi}{2}-\frac{\alpha}{2}\right)+\mathcal{O}(|\tilde{s}|) \\
\geq \sqrt{|\tilde{s}|} \sin (\alpha)+\mathcal{O}(|\tilde{s}|) \geq \frac{\eta}{K} \sqrt{|\tilde{s}|}(1+\mathcal{O}(\sqrt{|\tilde{s}|})) .
\end{array}
$$




\subsection{Proof of theorem 3 for interior order 2}

The first three rows of (4.3) are effected by the penalty. We have

$$
\begin{aligned}
& \tilde{s} \hat{\varepsilon}_{0}=i\left(\hat{\varepsilon}_{0}-2 \hat{\varepsilon}_{1}+\hat{\varepsilon}_{2}\right)+3 i \hat{\varepsilon}_{0}-h^{3} \hat{T}_{0}^{(1)}, \\
& \tilde{s} \hat{\varepsilon}_{1}=i\left(\hat{\varepsilon}_{0}-2 \hat{\varepsilon}_{1}+\hat{\varepsilon}_{2}\right)-2 i \hat{\varepsilon}_{0}, \\
& \tilde{s} \hat{\varepsilon}_{2}=i\left(\hat{\varepsilon}_{1}-2 \hat{\varepsilon}_{2}+\hat{\varepsilon}_{3}\right)+\frac{i}{2} \hat{\varepsilon}_{0} .
\end{aligned}
$$

For the other rows, $k=3,4, \ldots,(4.4)$ is valid. The boundary truncation error is $\hat{T}_{0}^{(1)}=i \hat{U}_{x x x}(0, s)+\mathcal{O}(h)=i s \hat{U}_{x}(0, s)+\mathcal{O}(h)$. Note that the $\mathcal{O}(h)$ part can be estimated together with the interior error, and will be omitted below.

Away from the boundary the solution is

$$
\hat{\varepsilon}_{j}=\sigma \kappa_{1}^{j-2}, j=2,3 \ldots
$$

where $\kappa_{1}$ is given by (4.10). The coefficient $\sigma$ and the remaining unknowns in the solution, $\hat{\varepsilon}_{0}$ and $\hat{\varepsilon}_{1}$, are determined from (4.14), which gives

$$
\underbrace{\left[\begin{array}{ccc}
-\kappa_{1}^{2} & -i \tilde{s}-4 & 2 \\
-\kappa_{1}^{2} & 1 & -i \tilde{s}+2 \\
(-i \tilde{s}+2) \kappa_{1}^{2}-\kappa_{1}^{3} & -0.5 & -1
\end{array}\right]}_{C(\tilde{s})} \underbrace{\left[\begin{array}{c}
\sigma \\
\hat{\varepsilon}_{0} \\
\hat{\varepsilon}_{1}
\end{array}\right]}_{\Sigma}=\left[\begin{array}{c}
-h^{3} i s \hat{U}_{x}(0, s) \\
0 \\
0
\end{array}\right] .
$$

We note that

$$
C(0)=\left[\begin{array}{ccc}
-1 & -4 & 2 \\
-1 & 1 & 2 \\
1 & -0.5 & -1
\end{array}\right]
$$

is non-singular. By the continuity of $C(\tilde{s})$ it follows that there exists a $\delta>0$ such that $\left|C(\tilde{s})^{-1}\right| \leq 2\left|C(0)^{-1}\right| \equiv K_{c}$ for all $|\tilde{s}| \leq \delta$ with $\operatorname{Re}(\tilde{s}) \geq 0$ (This claim can be generalized to other constants). We can now apply theorem 2.3, and (4.8) follows for $m=1$.

To show (4.9) we need to consider

$$
\|\hat{\varepsilon}\|_{h}^{2}=\sigma^{2} \sum_{0}^{\infty} h|\kappa(\tilde{s})|^{2}=\frac{h \sigma^{2}}{1-|\kappa(\tilde{s})|^{2}},
$$

for $\operatorname{Re}(\tilde{s}) \geq h^{2} \eta>0$. We assume $h$ is so small that $h^{2} K_{s} \leq \delta$, which is the case in our numerical study, and that $\left|C(\tilde{s})^{-1}\right| \leq 2\left|C(0)^{-1}\right|$ is valid for all relevant $\tilde{s}$. Then we can solve for $\Sigma$ and have

$$
|\sigma| \leq K_{c} h^{3}|s| \max _{0 \leq x \leq h}\left|\hat{U}_{x}(x, s)\right|
$$


By corollary 4.3 we have

$$
\|\hat{\varepsilon}\|_{h}^{2} \leq K \frac{h^{7}|s|^{2}}{\eta \sqrt{|\tilde{s}|}} \max _{0 \leq x \leq h}\left|\hat{U}_{x}(x, s)\right|^{2} \leq h^{6} \frac{\tilde{K}}{\eta} \max _{0 \leq x \leq h}\left|\hat{U}_{x}(x, s)\right|^{2},
$$

for any $\eta>0$. By Parseval's relation we have

$$
\begin{array}{r}
\int_{0}^{\infty} e^{-2 \eta t}\|\varepsilon\|_{h}^{2} d t=\frac{1}{2 \pi} \int_{-\infty}^{\infty}\|\hat{\varepsilon}(\cdot, \eta+i \xi)\|_{h}^{2} d \xi \\
\leq h^{6} \frac{K}{2 \pi \eta} \int_{-\infty}^{\infty} \max _{0 \leq x \leq h}\left|\hat{U}_{x}(x, \eta+i \xi)\right|^{2} d \xi \\
=h^{6} \frac{K}{\eta} \int_{0}^{\infty} e^{-2 \eta t} \max _{0 \leq x \leq h}\left|U_{x}(x, t)\right|^{2} d t
\end{array}
$$

Let $\eta=1 / t_{0}$ for some $t_{0}>0$. By arguing that the future cannot effect the past the upper limit of both time integrals can be replaced by $t_{0}$ and we obtain (4.9), with $K_{3}^{(1)} \sim \sqrt{t_{0} \int_{0}^{t_{0}}\left\|U_{x}\right\|^{2} d t}$. Thus we have shown that the boundary error is of order 3 .

\subsection{Proof of theorem 3 for interior order 4}

Since $p=4$, the four first equations of (4.1) have truncation errors of order $m=2$. Corresponding to (4.3) we have

$$
\begin{aligned}
& \tilde{s} \hat{\varepsilon}_{0}=i\left(2 \hat{\varepsilon}_{0}-5 \hat{\varepsilon}_{1}+4 \hat{\varepsilon}_{2}-\hat{\varepsilon}_{3}\right)+\frac{88 i}{17} \hat{\varepsilon}_{0}+i h^{4} \hat{T}_{0}^{(2)} \\
& \tilde{s} \hat{\varepsilon}_{1}=i\left(\hat{\varepsilon}_{0}-2 \hat{\varepsilon}_{1}+\hat{\varepsilon}_{2}\right)-\frac{144 i}{59} \hat{\varepsilon}_{0}+i h^{4} \hat{T}_{1}^{(2)} \\
& \tilde{s} \hat{\varepsilon}_{2}=i\left(-\frac{4}{43} \hat{\varepsilon}_{0}+\frac{59}{43} \hat{\varepsilon}_{1}-\frac{110}{43} \hat{\varepsilon}_{2}+\frac{59}{43} \hat{\varepsilon}_{3}-\frac{4}{43} \hat{\varepsilon}_{4}\right)+\frac{72 i}{43} \hat{\varepsilon}_{0}+i h^{4} \hat{T}_{2}^{(2)} \\
& \tilde{s} \hat{\varepsilon}_{3}=i\left(-\frac{1}{49} \hat{\varepsilon}_{0}+\frac{59}{49} \hat{\varepsilon}_{2}-\frac{118}{49} \hat{\varepsilon}_{3}+\frac{64}{49} \hat{\varepsilon}_{4}-\frac{4}{49} \hat{\varepsilon}_{5}\right)-\frac{16 i}{49} \hat{\varepsilon}_{0}+i h^{4} \hat{T}_{3}^{(2)}
\end{aligned}
$$

where after Taylor expansion we have

$$
\hat{T}_{B}^{(2)} \equiv\left(\begin{array}{c}
\hat{T}_{0}^{(2)} \\
\hat{T}_{1}^{(2)} \\
\hat{T}_{2}^{(2)} \\
\hat{T}_{3}^{(2)}
\end{array}\right)=\left(\begin{array}{c}
\frac{11}{12} \hat{U}_{x x x x}(0, s) \\
-\frac{1}{12} \hat{U}_{x x x x}(0, s) \\
\frac{5}{516} \hat{U}_{x x x x}(0, s) \\
\frac{11}{588} \hat{U}_{x x x x}(0, s)
\end{array}\right)+h \underbrace{\left(\begin{array}{c}
\hat{U}_{x x x x x}(0, s) \\
0 \\
0 \\
-\frac{1}{49} \hat{U}_{x x x x x}(0, s)
\end{array}\right)}_{\hat{\bar{T}}_{B}^{(2)}}+\mathcal{O}\left(h^{2}\right) .
$$

Note that in this case the leading term vanishes and the last term in (4.16) is of the same order as the interior truncation error, and can be estimated togeher with it. 
Away from the boundary we need to include both admissible roots (see lemma 4.2) to express the solution as

$$
\hat{\varepsilon}_{j}(\tilde{s})=\sigma_{1} \kappa_{1}^{j-2}(\tilde{s})+\sigma_{2} \kappa_{2}^{j-2}(\tilde{s}), \quad j=2,3, \ldots,
$$

and get a system of equations for $\hat{\varepsilon}_{0}, \hat{\varepsilon}_{1}, \sigma_{1}$ and $\sigma_{2}$. At $\tilde{s}=0$ we express $\hat{\varepsilon}_{2}(0), \hat{\varepsilon}_{3}(0), \varepsilon_{4}(0)$, and $\hat{\varepsilon}_{5}(0)$ in terms of $\sigma_{1}$ and $\sigma_{2}$. From (4.15) we get

$$
\underbrace{\left[\begin{array}{cccc}
-3 & \kappa_{2}^{(0)}-4 & -\frac{122}{17} & 5 \\
-1 & -1 & \frac{85}{59} & 2 \\
\frac{55}{43} & \frac{1}{43}\left(4\left(\kappa_{2}^{(0)}\right)^{2}-59 \kappa_{2}^{(0)}+110\right) & -\frac{68}{43} & -\frac{59}{43} \\
-\frac{1}{49} & \frac{1}{49}\left(4\left(\kappa_{2}^{(0)}\right)^{3}-64\left(\kappa_{2}^{(0)}\right)^{2}+118 \kappa_{2}^{(0)}-59\right) & 1 & 0
\end{array}\right]}_{C(0)}\left[\begin{array}{c}
\sigma_{1} \\
\sigma_{2} \\
\hat{\varepsilon}_{0} \\
\hat{\varepsilon}_{1}
\end{array}\right]=h^{5} \hat{\bar{T}}_{B}^{(2)} \text {. }
$$

Using lemma 4.2 we have

$$
C(0)=\left[\begin{array}{cccc}
-3 & 3-4 \sqrt{3} & -\frac{122}{17} & 5 \\
-1 & -1 & \frac{85}{59} & 2 \\
\frac{55}{43} & \frac{85}{43}+\frac{12}{43} \sqrt{3} & -\frac{68}{43} & -\frac{59}{43} \\
-\frac{1}{49} & -\frac{37}{49}-\frac{8}{49} \sqrt{3} & 1 & 0
\end{array}\right]
$$

which is is non-singular. Now (4.8) and (4.9) follow as in the $m=1$ case. The boundary error is here of order 5 .

\subsection{Proof of theorem 3 for interior order 6}

Following exactly the same approach we find that the solution of (4.3) is of the form

$$
\hat{\varepsilon}_{j}(\tilde{s})=\sigma_{1} \kappa_{1}^{j-3}(\tilde{s})+\sigma_{2} \kappa_{2}^{j-3}(\tilde{s})+\sigma_{3} \kappa_{3}^{j-3}(\tilde{s}), \quad j=3,4, \ldots,
$$

and from (4.3) we have a linear system for $\hat{\varepsilon}_{0}, \hat{\varepsilon}_{1}, \hat{\varepsilon}_{2}, \sigma_{1}, \sigma_{2}$ and $\sigma_{3}$. We get

$$
C(\tilde{s}) \Sigma=h^{5} \hat{T}_{B}^{(3)}, \quad \Sigma=\left[\sigma_{1} \sigma_{2} \sigma_{3} \hat{\varepsilon}_{0} \hat{\varepsilon}_{1} \hat{\varepsilon}_{2}\right]^{T},
$$

where $C(0)$, given in appendix $\mathrm{B}$, is non-singular. The truncation error in this case is to leading order $\left\|\hat{T}_{B}^{(3)}\right\| \sim\left|U_{x x x x x}(0, s)\right|$, which does not vanish at the boundary. Therefore the boundary error will be of order $h^{5}$. We can, as above, use the equation to express the truncation error in terms of time derivatives, enabling (4.8) and (4.9) follow as in the $m=1,2$ cases.

\section{Numerical experiments}

In this section numerical experiments are carried out to corroborate our theoretical results. We consider how the accuracy of the solution is affected by the lower order of accuracy near the boundaries. 
In the analysis we consider half-plane problems with only one boundary condition. For the purpose of numerical simulations boundary conditions need to be imposed on all computational boundaries. In the onedimensional setting with $N$ grid points, the penalty contribution, corresponding to weakly implemented Dirichlet conditions, in equation (3.5) is

$$
-\tau P^{-1} S^{T}\left(E_{0} u-E_{N-1} u\right), \quad \text { with } \tau=i .
$$

Corresponding boundary conditions are imposed in the $y$-dimension in the two-dimensional case.

We discretize in time, using an exponential integrator. The basic idea is to exploit that there exists an exact solution to the semi-discrete Schrödinger equation (3.5) with weak boundary conditions (where $\tau=i$ ) and initial conditions $u\left(t_{0}\right)=u_{0}$, given by

$$
u(t)=e^{-i H\left(t-t_{0}\right)} u_{0}, \quad H=-Q+P^{-1} S^{T}\left(E_{0}-E_{N-1}\right) .
$$

This type of exponential expression can be used to propagate the Schrödinger equation in time. Instead of taking the full matrix exponential, a few important eigenvalues and eigenvectors of $H$ can be generated, and the relation between the solution at time $t+\Delta t$ and $t$ can be expressed as

$$
u(t+\Delta t)=e^{-i H \Delta t} u(t) \approx e^{-i V \Lambda V^{-1} \Delta t} u(t)=V e^{-i \Lambda \Delta t} V^{-1} u(t),
$$

where $\Lambda$ is a diagonal matrix with the generated eigenvalues on the diagonal and the corresponding eigenvectors constitute the columns of the matrix $V$. The last term of (5.1) is reasonable to evaluate numerically since $\Lambda$ is a diagonal matrix of modest size. In our numerical experiments the time-propagation is carried out according to the exponential propagator (5.1), where a smaller subspace of eigenvalues and eigenvectors are computed using the Lanczos algorithm. The size of the Krylov subspace is chosen adaptively in order to fulfill a given accuracy requirement, whereas the time step is fixed. Since we investigate the accuracy of the semi-discrete system, the temporal error should preferably be kept smaller than the error that arise from the spatial discretization. For details on the temporal discretization we refer to [13].

Remark: A central finite difference discretization with periodic boundary conditions leads to the corresponding spatial discretization matrix $Q$ being Hermitian. Thus, eigenvalues and eigenvectors of $Q$ can be computed with the Lanczos scheme. The weakly imposed boundary conditions affect the structure of the spatial discretization matrix so that $H$ is not Hermitian, and instead the Arnoldi iteration for general matrices could be used. However, by the coordinate transformation $\omega=P^{1 / 2} u$, the spatial discretization matrix for the corresponding equation in $\omega, P^{1 / 2} H P^{-1 / 2}$, is 
Hermitian, and we can compute with the Lanczos algorithm by solving for $\omega$. In two dimensions we have $P^{1 / 2}=P_{x}^{1 / 2} \otimes P_{y}^{1 / 2}$.

We perform numerical computations in one and two dimensions with a traveling Gaussian, similar to the experiment displayed in figure 1. Starting with the initial conditions

$$
u(x, 0)=e^{i k_{x}\left(x-x_{0}\right)-\left(x-x_{0}\right)^{2}}
$$

in one dimension, and

$$
u(x, y, 0)=e^{i k_{x}\left(x-x_{0}\right)-\left(x-x_{0}\right)^{2}+i k_{y}\left(y-y_{0}\right)-\left(y-y_{0}\right)^{2}}
$$

in two dimensions, the Gaussian is propagated until it reaches the boundary. In the free boundary case, an exact solution exist for this problem. However, since we wish to investigate how the reduced accuracy near the boundaries affects the convergence rate, we compute the error as the difference between consecutive solutions, where the grid size is reduced by a factor two.

The $l_{2}$-error and $l_{\infty}$-error of the difference between the consecutive numerical solutions computed with grid size $2 h$ and $h, u^{2 h}$ and $u^{h}$, where $u^{h}$ is projected onto the coarser grid, are in each set of experiments computed at some final time $T$ as

$$
\begin{aligned}
\left\|u^{2 h}-u^{h}\right\|_{l_{2}} & =\sqrt{2 h \sum_{j=0}^{N-1}\left|u_{j}^{2 h}-u_{j}^{h}\right|^{2},} \\
\left\|u^{2 h}-u^{h}\right\|_{\infty} & =\max _{0 \leq j \leq N-1}\left|u_{j}^{2 h}-u_{j}^{h}\right|,
\end{aligned}
$$

in one dimension and

$$
\begin{array}{r}
\left\|u^{2 h_{x}, 2 h_{y}}-u^{h_{x}, h_{y}}\right\|_{l_{2}}=\sqrt{2 h_{x} 2 h_{y} \sum_{i=0}^{N_{1}-1} \sum_{j=0}^{N_{2}-1}\left|u_{i j}^{2 h_{x}, 2 h_{y}}-u_{i j}^{h_{x}, h_{y}}\right|^{2}}, \\
\left\|u^{2 h_{x}, 2 h_{y}}-u^{h_{x}, h_{y}}\right\|_{\infty}=\max _{0 \leq i \leq N_{1}-1} \max _{0 \leq j \leq N_{2}-1}\left|u_{i j}^{2 h_{x}, 2 h_{y}}-u_{i j}^{h_{x}, h_{y}}\right|
\end{array}
$$

in two dimensions. Convergence rates are computed as

$$
q=-\log \left(\frac{\left\|u^{2 h}-u^{h}\right\|}{\left\|u^{4 h}-u^{2 h}\right\|}\right) / \log (2) .
$$

We have used initial data (5.2) with $k_{x}=10, x_{0}=0$ in the onedimensional computations and initial data (5.3) with $k_{x}=8, k_{y}=6$, $x_{0}=0, y_{0}=0$ in the two-dimensional computations, on the computational 
domains $[-6,6]$ and $[-6,6]^{2}$, respectively. All simulations are run until $T=0.25$ using the time step $\Delta t=1 \cdot 10^{-4}$.

The errors in the $l_{2^{-}}$and $l_{\infty}$-norm and the convergence rates are displayed in tables 1-4 for the one-dimensional experiments and in tables 5-8 for the two-dimensional experiments. The convergence rates in the onedimensional experiments behave as expected. In the second and fourth order cases, we expect second and fourth order convergence both in $l_{2}$ norm and maximum-norm, which we clearly get. For order six, the analysis predicts the boundary error to be of order five. The convergence rate in maximum-norm does display 5 th order convergence, whereas the $l_{2}$-error rate is closer to order 5.5. A possible reason for this is that the modes corresponding to the admissible root close to one when $\tilde{s}$ is small has not yet propagated and reached the whole domain. In that case the boundary error is only localized to a small portion of the domain and one half order of accuracy will be gained. We have not done the theoretical analysis in the 8th order case. However, the experiments suggest that we get the same pattern as for the lower orders. Here, the leading order term in the truncation error is of even order and disappears on the boundary. Thus, we expect to gain one additional order (with respect to the two orders already gained) so that we get 7 th order convergence, which we also get for $N=1601$, see table 4 . As in the 6 th order case, the convergence rate for the $l_{2}$-error is a half order higher than the rate for the $l_{\infty}$-error. By visualizing the error, we conclude that the reduction of the convergence rate in $l_{2}$-norm for $N=3201$, denoted by ${ }^{*}$ in table 4 , is due to accumulated round-off errors.

In the two-dimensional experiments, the convergence rates in the second and fourth order cases, displayed in tables 5 and 6 , behave as expected, i.e. we get second and fourth order convergence rate, respectively. In table 7 , the 6 th order experiments shows 5 th order convergence rate in $l_{2}$-norm and approaching 5th order from below in maximum-norm, cf. the onedimensional simulations in table 3 . For the 8th order simulations with $N \leq 801$, we see the expected 7 th order convergence in $l_{2}$-norm in table 8. We get 6 th order convergence rate in maximum-norm, which is one order lower than expected. However, for the finest grid, $N=1601$, the convergence rates decrease significantly. The lower convergence rate is due to dominating errors, approximately of order $10^{-7}$, localized at the corners. We believe these are caused by singularities due to the non-smooth boundary. The additional row in table 8 with $N=1601^{*}$ corresponds to the 8th order simulation with $N=1601$, where points closer than ten points away from a boundary have been excluded before computing the errors and convergence rates. Here, we can see that we get 6 th order convergence rate of the $l_{\infty}$-error if the boundary points are excluded. The $l_{2}$-error convergence rate on the other hand is influenced by accumulated round-off 
errors, and we see that the $l_{2}$-error convergence is not affected so much by the corner points. In realistic computations we expect the overall errors to be larger than the error from corners (of order $10^{-7}$ ), so that the corner errors will not play an important role.

\begin{tabular}{ccccc}
\hline$N$ & $\left\|u^{2 h}-u^{h}\right\|_{l_{2}}$ & $\left\|u^{2 h}-u^{h}\right\|_{l_{\infty}}$ & $q_{l_{2}}$ & $q_{l_{\infty}}$ \\
\hline 51 & - & - & - & - \\
101 & 1.51 & $9.60 \cdot 10^{-1}$ & - & - \\
201 & 1.87 & 1.45 & -0.30 & -0.60 \\
401 & $6.85 \cdot 10^{-1}$ & $6.73 \cdot 10^{-1}$ & 1.44 & 1.12 \\
801 & $1.78 \cdot 10^{-1}$ & $2.09 \cdot 10^{-1}$ & 1.94 & 1.69 \\
1601 & $4.48 \cdot 10^{-2}$ & $5.46 \cdot 10^{-2}$ & 1.99 & 1.93 \\
3201 & $1.12 \cdot 10^{-2}$ & $1.38 \cdot 10^{-2}$ & 2.00 & 1.99 \\
\hline
\end{tabular}

Table 1: Errors for 2nd order spatial discretization, one-dimensional simulations.

\begin{tabular}{ccccc}
\hline$N$ & $\left\|u^{2 h}-u^{h}\right\|_{l_{2}}$ & $\left\|u^{2 h}-u^{h}\right\|_{l_{\infty}}$ & $q_{l_{2}}$ & $q_{l_{\infty}}$ \\
\hline 51 & - & - & - & - \\
101 & 1.66 & $9.18 \cdot 10^{-1}$ & - & - \\
201 & $6.68 \cdot 10^{-1}$ & $7.09 \cdot 10^{-1}$ & 1.31 & 0.37 \\
401 & $5.04 \cdot 10^{-2}$ & $6.76 \cdot 10^{-2}$ & 3.73 & 3.39 \\
801 & $3.13 \cdot 10^{-3}$ & $4.13 \cdot 10^{-3}$ & 4.01 & 4.03 \\
1601 & $1.97 \cdot 10^{-4}$ & $2.63 \cdot 10^{-4}$ & 3.99 & 3.97 \\
3201 & $1.24 \cdot 10^{-5}$ & $1.66 \cdot 10^{-5}$ & 3.99 & 3.99 \\
\hline
\end{tabular}

Table 2: Errors for 4th order spatial discretization, one-dimensional simulations.

\begin{tabular}{ccccc}
\hline$N$ & $\left\|u^{2 h}-u^{h}\right\|_{l_{2}}$ & $\left\|u^{2 h}-u^{h}\right\|_{l_{\infty}}$ & $q_{l_{2}}$ & $q_{l_{\infty}}$ \\
\hline 51 & - & - & - & - \\
101 & 1.74 & $9.72 \cdot 10^{-1}$ & - & - \\
201 & $2.23 \cdot 10^{-1}$ & $2.75 \cdot 10^{-1}$ & 2.96 & 1.82 \\
401 & $4.24 \cdot 10^{-3}$ & $6.08 \cdot 10^{-3}$ & 5.71 & 5.50 \\
801 & $9.03 \cdot 10^{-5}$ & $3.28 \cdot 10^{-4}$ & 5.55 & 4.21 \\
1601 & $2.14 \cdot 10^{-6}$ & $1.28 \cdot 10^{-5}$ & 5.40 & 4.67 \\
3201 & $4.41 \cdot 10^{-8}$ & $4.23 \cdot 10^{-7}$ & 5.60 & 4.92 \\
\hline
\end{tabular}

Table 3: Errors for 6 th order spatial discretization, one-dimensional simulations. 


\begin{tabular}{ccccc}
\hline$N$ & $\left\|u^{2 h}-u^{h}\right\|_{l_{2}}$ & $\left\|u^{2 h}-u^{h}\right\|_{l_{\infty}}$ & $q_{l_{2}}$ & $q_{l_{\infty}}$ \\
\hline 51 & - & - & - & - \\
101 & 1.61 & 1.15 & - & - \\
201 & $4.50 \cdot 10^{-1}$ & $5.43 \cdot 10^{-1}$ & 1.83 & 1.08 \\
401 & $4.96 \cdot 10^{-3}$ & $1.06 \cdot 10^{-2}$ & 6.50 & 5.68 \\
801 & $3.84 \cdot 10^{-5}$ & $1.63 \cdot 10^{-4}$ & 7.01 & 6.02 \\
1601 & $2.10 \cdot 10^{-7}$ & $1.27 \cdot 10^{-6}$ & 7.51 & 7.00 \\
3201 & $4.52 \cdot 10^{-9}$ & $1.07 \cdot 10^{-8}$ & $5.54^{*}$ & 6.90 \\
\hline
\end{tabular}

Table 4: Errors for 8th order spatial discretization, one-dimensional simulations.

\begin{tabular}{ccccc}
\hline$N$ & $\left\|u^{2 h_{x}, 2 h_{y}}-u^{h_{x}, h_{y}}\right\|_{l_{2}}$ & $\left\|u^{2 h_{x}, 2 h_{y}}-u^{h_{x}, h_{y}}\right\|_{l_{\infty}}$ & $q_{l_{2}}$ & $q_{l_{\infty}}$ \\
\hline 51 & - & - & - & - \\
101 & 1.92 & 1.06 & - & - \\
201 & 1.53 & $9.60 \cdot 10^{-1}$ & 0.32 & 0.14 \\
401 & $4.47 \cdot 10^{-1}$ & $2.60 \cdot 10^{-1}$ & 1.78 & 1.89 \\
801 & $1.14 \cdot 10^{-1}$ & $6.47 \cdot 10^{-2}$ & 1.98 & 2.01 \\
\hline
\end{tabular}

Table 5: Errors for 2nd order spatial discretization, two-dimensional simulations.

\begin{tabular}{ccccc}
\hline$N$ & $\left\|u^{2 h_{x}, 2 h_{y}}-u^{h_{x}, h_{y}}\right\|_{l_{2}}$ & $\left\|u^{2 h_{x}, 2 h_{y}}-u^{h_{x}, h_{y}}\right\|_{l_{\infty}}$ & $q_{l_{2}}$ & $q_{l_{\infty}}$ \\
\hline 51 & - & - & - & - \\
101 & 1.96 & 1.14 & - & - \\
201 & $2.85 \cdot 10^{-1}$ & $1.64 \cdot 10^{-1}$ & 2.79 & 2.80 \\
401 & $1.94 \cdot 10^{-2}$ & $1.10 \cdot 10^{-2}$ & 3.87 & 3.90 \\
801 & $1.24 \cdot 10^{-3}$ & $7.01 \cdot 10^{-4}$ & 3.97 & 3.97 \\
\hline
\end{tabular}

Table 6: Errors for 4th order spatial discretization, two-dimensional simulations.

\begin{tabular}{ccccc}
\hline$N$ & $\left\|u^{2 h_{x}, 2 h_{y}}-u^{h_{x}, h_{y}}\right\|_{l_{2}}$ & $\left\|u^{2 h_{x}, 2 h_{y}}-u^{h_{x}, h_{y}}\right\|_{l_{\infty}}$ & $q_{l_{2}}$ & $q_{l_{\infty}}$ \\
\hline 51 & - & - & - & - \\
101 & 1.41 & $8.38 \cdot 10^{-1}$ & - & - \\
201 & $5.61 \cdot 10^{-2}$ & $3.51 \cdot 10^{-2}$ & 4.65 & 4.58 \\
401 & $1.03 \cdot 10^{-3}$ & $7.50 \cdot 10^{-4}$ & 5.76 & 5.55 \\
801 & $1.94 \cdot 10^{-5}$ & $4.32 \cdot 10^{-5}$ & 5.74 & 4.12 \\
1601 & $6.40 \cdot 10^{-7}$ & $1.66 \cdot 10^{-6}$ & 4.92 & 4.70 \\
\hline
\end{tabular}

Table 7: Errors for 6th order spatial discretization, two-dimensional simulations. 


\begin{tabular}{ccccc}
\hline$N$ & $\left\|u^{2 h_{x}, 2 h_{y}}-u^{h_{x}, h_{y}}\right\|_{l_{2}}$ & $\left\|u^{2 h_{x}, 2 h_{y}}-u^{h_{x}, h_{y}}\right\|_{l_{\infty}}$ & $q_{l_{2}}$ & $q_{l_{\infty}}$ \\
\hline 51 & - & - & - & - \\
101 & $9.75 \cdot 10^{-1}$ & $5.75 \cdot 10^{-1}$ & - & - \\
201 & $6.39 \cdot 10^{-2}$ & $6.15 \cdot 10^{-2}$ & 3.93 & 3.22 \\
401 & $7.35 \cdot 10^{-4}$ & $9.87 \cdot 10^{-4}$ & 6.44 & 5.96 \\
801 & $5.02 \cdot 10^{-6}$ & $1.69 \cdot 10^{-5}$ & 7.19 & 5.87 \\
1601 & $3.35 \cdot 10^{-7}$ & $1.71 \cdot 10^{-6}$ & 3.90 & 3.30 \\
\hline $1601^{*}$ & $3.17 \cdot 10^{-7}$ & $2.58 \cdot 10^{-7}$ & 3.99 & 6.03 \\
\hline
\end{tabular}

Table 8: Errors for 8th order spatial discretization, two-dimensional simulations.

In order to illustrate the efficiency in using higher order methods, table 9 displays the $l_{2}$-error obtained using different orders of spatial discretization with the same grid density, and the required number of grid points for respective order to obtain an error smaller than $10^{-2}$. For a fair comparison, the computational complexity should be considered for methods of different orders, as a denser structure of the spatial discretization matrix also affects the efficiency in the time-stepping. Figure 3 shows how the error depends on the CPU time. Clearly, as seen in table 9 and figure 3, it pays off to use higher order methods. In order to determine how the 8th order method compares to the 6th order method, it would be interesting to consider other aspects, such as the effect of local grid refinement and parallelization aspects.

\begin{tabular}{ccc}
\hline order & $l_{2}$-error $(\mathrm{N}=801)$ & Grid points $\left(l_{2}\right.$-error $\left.\leq 10^{-2}\right)$ \\
\hline 2 & $1.78 \cdot 10^{-1}$ & - \\
4 & $3.13 \cdot 10^{-3}$ & 801 \\
6 & $9.03 \cdot 10^{-5}$ & 401 \\
8 & $3.84 \cdot 10^{-5}$ & 401 \\
\hline
\end{tabular}

Table 9: Comparison of errors, one-dimensional simulations. 


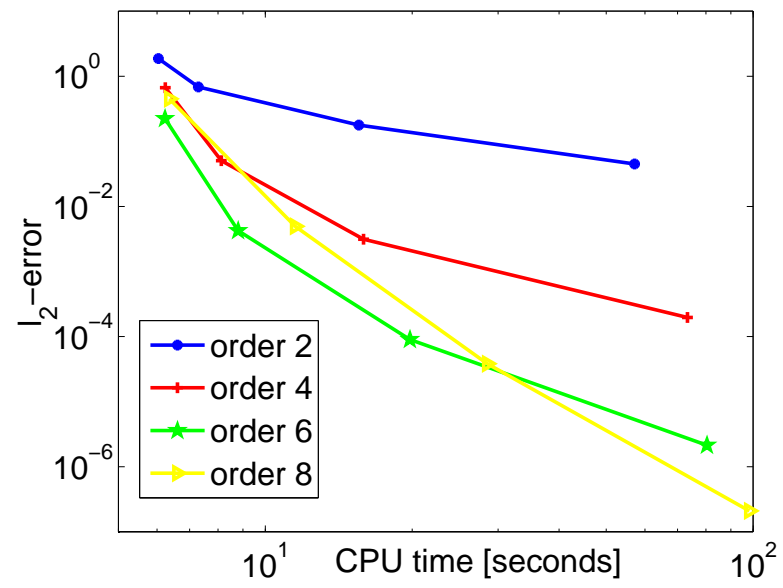

Figure 3: $l_{2}$-error as a function of CPU time for different order of spatial discretizations, one-dimensional simulations.

\section{Conclusions}

In this paper we extend the SBP-SAT framework to the Schrödinger equation. We derive stability conditions for the Schrödinger equation with homogeneous Dirichlet boundary conditions and analyze the accuracy of the method using normal mode analysis. For SBP operators of interior order $2 m$ with boundary stencils of order $m$, global accuracy of order $m+2$ is shown for $m \geq 2$ (global accuracy of order 2 for $m=1$ ).

The accuracy analysis is a general approach and can very well be used for other equations, for which a stability estimate exists. In the general case, for an equation of order $q$, where the boundary truncation error is of order $m$, we can hope for errors $\sim h^{q+m}$, see also [9].

The theoretical results of stability and accuracy are verified in numerical simulations. We conclude by the numerical experiments that higher order methods pays off even for one-dimensional problems of modest size. We expect that a corresponding comparison in higher dimensions would be even more advantageous for higher order methods.

An additional way to increase efficiency in the simulations is to include local grid refinement in the framework. Accuracy and stability at non-conforming grid interfaces for the Schrödinger equation, that allow for structured adaptive mesh refinement, is the topic of a forthcoming paper. 


\section{A Proof of lemma 2.1}

We want to prove that $w^{T} R w \geq \alpha h w_{1}^{2} \forall w^{T}=\left(w_{1}, w_{2}, \ldots\right)$ so that $R-$ $\alpha h E_{0} \geq 0$. We have

$$
R=h\left[\begin{array}{l|l|ll}
R_{0} & & \\
& & -1 & \\
\hline-1 & & A_{2}
\end{array}\right]
$$

where $R_{0}$ is a positive definite $3 \times 3$ matrix

$$
R_{0}=\left[\begin{array}{ccc}
\frac{4}{9} & -\frac{2}{9} & \frac{2}{9} \\
-\frac{2}{9} & \frac{10}{9} & -\frac{10}{9} \\
\frac{2}{9} & -\frac{10}{9} & \frac{19}{9}
\end{array}\right],
$$

and

$$
A_{2}=\left[\begin{array}{ccc}
2 & -1 & \\
-1 & 2 & -1 \\
& & \ddots
\end{array}\right]
$$

We will need that $\tilde{R}_{0}=R_{0}-E_{2}-\alpha E_{0} \geq 0$, for some $\alpha>0$, where

$$
E_{2}=\left[\begin{array}{lll}
0 & 0 & 0 \\
0 & 0 & 0 \\
0 & 0 & 1
\end{array}\right],
$$

$E_{0}=\operatorname{diag}(1,0, \ldots)$ and $\alpha$ should be determined. Furthermore, we write $A_{1}=A_{2}-E_{0}, A_{1} \geq 0$. Then we have

$$
y^{T} A_{2} y=y^{T} A_{1} y+y_{1}^{2} \geq y_{1}^{2} .
$$

Now let $w^{T}=\left(x^{T}, y^{T}\right)$, where $x$ has only three components while $y^{T}=$ $\left(y_{1}, y_{2}, \ldots\right)$. Then

$$
\begin{array}{r}
w^{T} R w=h\left(x^{T} R_{0} x-2 x_{3} y_{1}+y^{T} A_{2} y\right) \\
\geq h(x^{T} \tilde{R}_{0} x+\alpha x_{1}^{2}+\underbrace{x_{3}^{3}-2 x_{3} y_{1}+y_{1}^{2}}_{\left(x_{3}-y_{1}\right)^{2}}) \\
\geq \alpha h x_{1}^{2}=\alpha h w_{1}^{2} .
\end{array}
$$


In order to determine $\alpha$, we compute the eigenvalues $\lambda_{i}, i=1,2,3$, of $\tilde{R}_{0}$, where $\lambda_{1}=0, \lambda_{2,3}=-\frac{\alpha-\frac{24}{9}}{2} \pm \sqrt{\left(\frac{\alpha-\frac{24}{9}}{2}\right)^{2}+\frac{4}{9}\left(5 \alpha-\frac{18}{9}\right)}$. We see from the expression under the square root that $\tilde{R}_{0}$ is positive semi-definite for $\alpha \leq \frac{18}{45}=0.4$. Thus, we have proved lemma 2.1 with $\alpha=0.4$.

\section{B $C(0)$ for Dirichlet boundary conditions, or- der 6}

The matrix $\mathrm{C}(0)$ for Dirichlet boundary conditions, order 6 , is given by

$C(0) \approx\left[\begin{array}{cccccc}2.9794 & 3.3686-0.02305 i & 3.3686+0.02305 i & -9.3821 & 8.0245 & -8.2157 \\ 0.5705 & 1.0025-0.04695 i & 1.0025+0.04695 i & 1.8235 & 2.3540 & -1.8675 \\ -4.6151 & -6.7899+0.2266 i & -6.7899-0.2266 i & -4.1363 & -4.1376 & 8.1084 \\ 2.5660 & 4.5927-0.1966 i & 4.5927+0.1966 i & 0.8615 & 1.1591 & -3.5117 \\ -1.2510 & -3.1883+0.2835 i & -3.1883-0.2835 i & -0.09514 & -0.9157 & 1.9876 \\ 0.1996 & 0.3281-0.1029 i & 0.3281+0.1029 i & -0.04002 & 0.1875 & -0.3471\end{array}\right]$,

and is non-singluar.

\section{SBP operators, order 2}

Below are the SBP operators, $Q, P, A$, and $S$ for the 2nd order discretization, where $Q=P^{-1}\left(-A-E_{0} S\right)$.

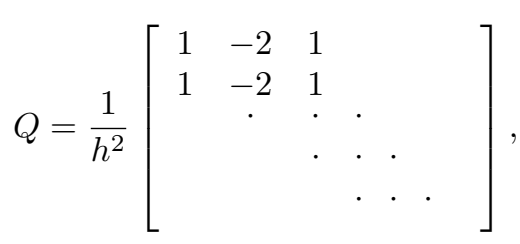

$$
\begin{aligned}
& P=h\left[\begin{array}{ccccc}
\frac{1}{2} & & & & \\
& 1 & & \\
& & \cdot & \\
& & & \cdot \\
& & & \cdot & \\
& & & &
\end{array}\right],
\end{aligned}
$$




$$
\begin{aligned}
& A=\frac{1}{h}\left[\begin{array}{cccccc}
1 & -1 & & & \\
-1 & 2 & -1 & & \\
& \cdot & \cdot & \cdot & \\
& & \cdot & \cdot & \\
& & & \cdot & \cdot
\end{array}\right], \\
& S=\frac{1}{h}\left[\begin{array}{ccccc}
-\frac{3}{2} & 2 & -\frac{1}{2} & & \\
& 1 & \cdot & & \\
& & & \cdot &
\end{array}\right] .
\end{aligned}
$$




\section{References}

[1] M. Born, R. Oppenheimer. (1927). Zur Quantentheorie der Molekeln, Ann. Phys. 84, 457-484.

[2] D. Kosloff, R. Kosloff. (1983). A Fourier method solution for the time dependent Schrödinger equation as a tool in molecular dynamics, J. Comput. Phys. 52, 35-53.

[3] M. H. Carpenter, D. Gottlieb, S. Abarbanel. (1994). Time-stabe boundary conditions for finite difference schemes solving hyperbolic systems: Methodology and application to high-order compact schemes. J. Comput. Phys. 111, 220-236.

[4] H-O. Kreiss, G. Scherer. (1974). Finite element and finite difference methods for hyperbolic partial differential equations, In: Mathematical aspects of finite elements in partial differential equations, Academic Press, New York.

[5] B. Strand. (1994). Summation by parts for finite difference approximations for d/dx, J. Comput. Phys. 110, 47-67.

[6] M. H. Carpenter, J. Nordström, D. Gottlieb. (1999). A stable and conservative interface treatment of arbitrary spatial accuracy, J. Comput. Phys. 148, 341-365.

[7] B. Gustafsson. (1975). The convergence rate for difference approximations to mixed initial boundary value problems, Math. Comp. 29, 396-406.

[8] B. Gustafsson. (1981). The convergence rate for difference approximations to general mixed initial boundary value problems, SIAM J. Numer. Anal. 18, 179-190.

[9] M. Svärd, J. Nordström. (2006). On the order of accuracy for difference approximations of initial-boundary value problems, J. Comput. Phys. $218,333-352$.

[10] B. Gustafsson, H-O. Kreiss and J. Oliger. (1995). Time-dependent problems and difference methods, John Wiley \& Sons, Inc., New York.

[11] K. Mattsson, J. Nordström. (2004). Summation by parts operators for finite difference approximations of second derivatives, J. Comput. Phys. 199, 503-540.

[12] C. E. Baumann, J. T. Oden. (1999). A discontinuous $h p$ finite element method for convection-diffusion problems, Comput. Methods Appl. Mech. Engrg. 175, 311-341. 
[13] K. Kormann, S. Holmgren and H. O. Karlsson. (2008). Accurate time propagation for the Schrödinger equation with an explicitly timedependent Hamiltonian, J. Chem Phys., 128, 184101:1-11. 